\title{
Essais
}

Revue interdisciplinaire d'Humanités

$17 \mid 2021$

Quels lieux pour les morts?

\section{Envers la vie et la mort : passage de la Chine au Japon chez Claudel}

Towards life and death: Claudel's passage from China to Japan

\section{Yi Xu}

\section{OpenEdition}

\section{Journals}

Édition électronique

URL : https://journals.openedition.org/essais/8994

DOI : 10.4000/essais.8994

ISSN : 2276-0970

Éditeur

École doctorale Montaigne Humanités

Édition imprimée

Date de publication : 15 avril 2021

Pagination : 137-146

ISBN : 979-10-97024-09-3

ISSN : $2417-4211$

Référence électronique

Yi Xu, "Envers la vie et la mort: passage de la Chine au Japon chez Claudel », Essais [En ligne], 17 | 2021, mis en ligne le 27 avril 2021, consulté le 18 janvier 2023. URL : http://journals.openedition.org/ essais/8994; DOI : https://doi.org/10.4000/essais.8994 


\section{Envers la vie et la mort: passage de la Chine au Japon chez Claudel}

La réflexion sur la vie et la mort est un des éléments fondamentaux de l'univers poétique, dramatique et même métaphysique de Paul Claudel. Il fait la confidence, dans $\mathrm{Ma}$ Conversion, que la mort lui a inspiré « une profonde terreur et la pensée de la mort ne [le] quittait pas. " Beaucoup d'études, telles celles de Pierre Brunel ${ }^{2}$ ou de Gilbert Viprey ${ }^{3}$, ont abordé ce sujet de manière convaincante dans le cadre de ses drames; d'autres critiques ont éclairé les principaux aspects liés à la conception de la mort par l'approche des croyances ou de l'esthétique de l'Extrême-Orient : c'est le cas dans les études de Gilbert Gadoffre ${ }^{4}$ et de Michel Malicet ${ }^{5}$. Guidée par ces recherches, nous nous proposons d'apporter quelques précisions sur la notion de la " mort » et de ses lieux chez l'écrivain-voyageur, en étudiant quelques-unes des images de ses poèmes écrits en Chine et au Japon.

\section{Chez les morts : du paysage au rite}

Si la mort dans le théâtre de Claudel est marquée par « une vision brutale et assez macabre de décomposition et de pourriture $"^{6}$, surtout dans un de ses premiers drames, Tête d'Or, il en est de même à peu près cinq ans plus tard pour ses premiers poèmes en prose pendant son séjour diplomatique dans

1 Paul Claudel, Euvre en prose, Paris, Gallimard, 1973, p. 1009.

2 Pierre Brunel, L'Évocation des morts et la descente aux enfers. Homère, Virgile. Dante. Claudel, Paris, SEDES, 1974.

3 Gilbert Viprey, «Images de la mort », in Jacques Petit (éd.), Paul Claudel, 3 : Thèmes et images, Paris, Lettres modernes, 1966, p. 28-37.

4 Gilbert Gadoffre, Claudel et l'univers chinois, Paris, Gallimard, 1968.

5 Michel Malicet, P.C., le Poète et le Shamisen. Le poìte et le vase d'encens. Jules ou l'homme-auxdeux-cravates, Édition critique et commentée, Paris, Les Belles Lettres, 1970.

6 Gilbert Viprey, "Images de la mort », in Jacques Petit (éd.), Paul Claudel, 3 : Thèmes et images, op. cit., p. 28-29. 
l'Empire du Milieu. Le monde des morts en 1896 est en général un "vaste " espace, isolé du monde des vivants et caractérisé par une sorte de saleté asiatique, où sont "partout des cercueils, des monticules couverts de roseaux flétris [...] des rangées de petits pieux en pierre, des statues mitrées, des lions, indiquant les sépultures antiques [...] ». La salle "ténébreuse " de la pagode ou du temple " au loin entre les bosquets de bambous " ${ }^{7}$ renferme " un démon brun à quatre paires de bras, la face convulsée par la rage » qui «s'y tient caché comme un assassin ${ }^{8}$. De plus, l'effroi est alourdi par une description violente et réaliste des misères de l'être humain : à côté " des édifices entourés d'arbres et de haies " et bâtis par "les corporations, les riches ", se trouve un "puits rempli de cadavres de petites filles dont leurs parents se sont débarrassés. On l'a bouché, une fois comble; il en faudra creuser un autre »".

Il est à remarquer que malgré l'effet de distance créé entre l'espace des vivants et celui des morts, Claudel atteste en même temps, fidèle à la réalité, que les "décombres et ces jachères " sont " dans une même enceinte juxtaposés aux multitudes les plus denses ", et qu' "à côté de minutieuses cultures " se localisent "ces monts stériles et l'étendue infinie des cimetières ${ }^{10}$. Il semble que cette juxtaposition, cette coexistence, et même cette harmonie curieuse de paradoxes occupent une grande importance chez Claudel. Le titre du poème "Tombes.-Rumeurs» en fait un bon exemple. De plus, en suivant les lignes de ce poème, nous pouvons facilement reconnaître que le monde de la décomposition et du pourrissement n'est pas quelque chose de purement négatif, et que l'isolement n'est jamais absolu : "Le défunt, dès qu'il a trépassé, devient une chose importante et suspecte, un protecteur malfaisant, - morose, quelqu'un qui est là et qu'il faut se concilier. Les liens entre les vivants et les morts se dénouent mal, les rites subsistent et se perpétuent. $"{ }^{11}$ En effet, c'est précisément le culte des morts pratiqué en Chine comme d'ailleurs dans toute l'Asie qui a frappé Claudel, et « ce thème profond de la continuité malgré la séparation apparente $»^{12}$ sera repris non seulement dans plusieurs poèmes composés en Chine, "Fête des morts le septième mois ", "Banyan " par exemple, mais aussi au Japon dans l'observation de la cérémonie en l'honneur des victimes du séisme 1923, des funérailles de l'empereur Yoshi-hito, ainsi que dans l'inspiration du texte " Le Poète et le vase d'encens".

7 Paul Claudel, Euvre poétique, Paris, Gallimard, 1967, p. 26-27.

8 Ibid., p. 30.

9 Ibid., p. 27.

10 Ibid., p. 77-78.

11 Ibid., p. 42.

12 Henri Micciollo, L'Oiseau noir dans le soleil levant de Paul Claudel. Introduction, variantes et notes, Paris, Les Belles Lettres, 1980, p. 188. 


\section{Éclat des flammes en Chine}

En Chine, le culte des morts s'effectue à l'aide de plusieurs réalités, parmi lesquelles celle du brûlage contient la valeur la plus suggestive pour l'écrivain-voyageur à l'égard de son initiation à la sagesse orientale. Thème courant chez Claudel, le feu, tandis qu'il apporte la destruction à tant de personnages sous une forme soit "violente " (incendie, explosion), soit " suggérée "13 (le coucher du soleil), se transforme d'abord en flammes rassurant les âmes séparées dans les deux mondes. Il faut surtout lire la description des flammes de toutes formes dans la nuit de la fête annuelle où se rejoignent les vivants et les revenants :

La flute guide les âmes, le coup du gong les rassemble comme des abeilles. Dans les noires ténèbres, l'éclat de la flamme les apaise et les rassasie. [...] [À] la proue le brûlot flamboyant, au mât le feston ballotté des lanternes, rehausse d'une touche ardente l'air éteint, comme dans une chambre spacieuse une chandelle que l'on tient au poing éclaire le vide solennel de la nuit. Cependant, le signal est donné ; les flûtes éclatent, le gong tonne, les pétards pètent, les trois bateliers s'attellent à la longue godille. La barque part et vire, laissant dans le large mouvement de son sillage une file de feux : quelqu'un sème de petites lampes. Lueurs précaires, sur la vaste coulée des eaux opaques, cela clignote un instant et périt. Un bras saisissant le flambeau d'or, la botte de feu qui fond et flamboie dans la fumée, en touche le tombeau des eaux : l'éclat illusoire de la lumière, tel que des poissons, fascine les froids noyés. D’autres barques illuminées vont et viennent ; on entend au loin des détonations, et sur les bateaux de guerre deux clairons, s'enlevant l'un à l'autre la parole, sonnent ensemble l'extinction des feux. ${ }^{14}$

Comme l'a confirmé Gilbert Gadoffre, ce poème intitulé " Fête des morts le septième mois ", ne permettant que "d'entrevoir fragmentairement ${ }^{15}$ la cérémonie, est terminé en février 1896, tandis que le rituel auquel Claudel a assisté a dû s'effectuer à la fin d'août de l'année précédente. Autrement dit, la célébration concernant les Lanternes flottantes, dont il connaît peu pour le moment le sens bouddhique et taö̈ste, pourrait constituer sa première initiation aux coutumes chinoises dès son arrivée en juillet 1895 .

D'abord, l'attente du départ est marquée par les flammes en un double sens. D'un côté, le "brûlot flamboyant " à la proue, "la touche ardente " et les " lanternes » du feston ballotté servent à éclairer le noir du ciel et de la rivière ; de l'autre, l'éclat de la flamme apaise et rassasie la foule. En effet, ce double sens évocateur et protecteur correspond bien à la signification tradi-

13 Gilbert Viprey, "Images de la mort », in Jacques Petit (éd.), Paul Claudel, 3 : Thèmes et images, op. cit., p. 29.

14 Paul Claudel, Euvre poétique, op. cit., p. 36.

15 Gibert Gadoffre, Paul Claudel ; éd. critique avec introduction, variantes et commentaire par Gilbert Gadoffre, Paris, Mercure de France, 1973, p. 108. 
tionnelle de l'action de brûler en Extrême-Orient : d'une part, le brûlage, avec ses lumières et ses fumées, assure l'envoi des offrandes aux défunts; d'autre part, il constitue une frontière entre les vivants et les morts, de sorte que les parents de ces derniers ne regardent pas en arrière. D'où vient que l'angoisse puisse être « apaisée et rassasiée » par les flammes, où se reflètent à la fois l'attachement et la considération des vivants vers leurs chers décédés.

Ensuite, le processus du rituel est marqué par la rapide communication entre les flammes et la rivière avec la touche saisissante du poète, qui, sans doute, intensifie des sens plus riches que ceux d'" une coulée homogène ", résumé par G. Gadoffre. ${ }^{16}$ "De petites lampes " semées dans la rivière composent " une file de feux ", dont la lumière faible ne dure qu'à " clignoter " un instant. Le feu remplit ici une fonction médiatrice, surtout dans le contexte où Claudel métamorphose "la vaste coulée des eaux " en " tombeau ". Si la diffusion des fumées remontant au ciel est lente et successive, "l'éclat de la lumière » de «la botte du feu » est "illusoire » et fugitif, une fois communiqué à ce " tombeau des eaux ". Pourquoi « clignoter " ? Pourquoi « illusoire " ? À part le fait naturel que le feu s'éteint assez vite dans l'eau, il se pourrait que le feu, symbole de l'énergie vitale, meurt souvent à une vitesse qui nous laisse peu de temps pour saisir quand cela a eu lieu, et où. De plus, " toucher » est un verbe de communication, lié au sens humain. Ainsi, nous pouvons dire que la description est comme une esquisse symbolisant la «limite des deux mondes indécise ${ }^{17}$, thème largement développé chez Claudel à la suite de ses lectures sur les doctrines taoïstes. Les flammes évocatrices, médiatrices et protectrices éclatent donc aussi pour inspirer à l'ermite claudélien sa future conception du yin-yang comme transformation. Dans sa conférence sur les superstitions chinoises donnée en 1910, l'année suivant son retour définitif de Chine, Claudel dit :

Ce cercle formé de l'accolement tête-bêche de deux espèces de têtards, l'un blanc, l'autre noir, représente la conjonction des deux principes opposés dont les éternelles transformations constituent l'évolution universelle. Le Yang représente le blanc, le Yin le noir, le premier le plein, l'autre le vide, l'un le chaud, l'autre le froid, l'un la terre, l'autre le ciel, l'un le relief, l'autre le creux, l'un le mâle, l'autre la femelle, etc. [...] Chacune porte en soi le germe de l'autre.

Pour Claudel catholique, le monde de l'Au-delà désigne traditionnellement le lieu où Dieu et les âmes des morts résident. Loin du sens chrétien de la rédemption et de la délivrance, la vie et la mort en Chine, sans Dieu, se transforment par le cercle "roulant sans frottement et sans déchet " selon l'ancienne sagesse orientale. L'espace des morts est plutôt une "vie future " « en vide " où se trouvent « les mêmes mœurs, les mêmes habitudes, le même

16 Ibid., p. 108.

17 Gilbert Gadoffre, Claudel et l'univers chinois, op. cit., p. 345. 
gouvernement, la même administration » que dans le monde des vivants. Un espace "spirituel " superposé au monde " matériel " dont " les frontières demeurent parfois incertaines et mal fixées ${ }^{18}$. L'indécision de la frontière entre la vie et la mort conduit sans doute, dans les années suivantes, à l'enrichissement optimiste des éléments dans l'écriture du paysage sépulcral.

Par exemple : le poète éprouve une joie de vivre et la paix interieure dans le poème " La navigation nocturne » en 1897 : « Je vogue en paix au travers de la région modérée [...] À ce douzième mois encore, cimetière et potager, la campagne, avec les tertres partout des tombes, s'étend productive et funèbre. Les bosquets de bambous bleus, les pins sombres au-dessus des sépultures, les roseaux glauques, arrêtent avec art le regard en le satisfaisant $[\ldots]{ }^{19}$. Notons aussi la prospérité qui coexiste avec les morts dans «Le point » publié en 1903 : « [...] j'envisage le pays des morts. Avec ses bouquets de pins et d'oliviers, il se disperse et s'épand au milieu des profondes moissons qui l'entourent. ${ }^{20}$ Encore plus loin, lors de sa visite au mausolée d'Ieyasu au Japon en 1898, voici une vision toute proche de celle du cercle yin-yang : "L'Averne devant nous s'ouvre et se déploie. Son sol brûlé, ce ciel bas, cette amère clôture de volcans et de sapins, ne correspondent-ils pas à ce fond noir et nul sur lequel se lèvent les visions des songes? Ainsi, avec une sagesse royale, l'antique shogun Ieyasu choisit ce lieu pour en superposer à l'ombre qu'il réintègre les ombrages, et, par la dissolution de son silence dans leur opacité, opérer la métamorphose du mort dans un dieu, selon l'association d'un temple à la sépulture. $»^{21}$

\section{Les Japonais dans les flammes}

Le retour de Claudel au Japon comme ambassadeur a été très vite marqué par le grand séisme du Kantō le $1^{\text {er }}$ septembre 1923. Cette catastrophe a détruit Yokohama et Tokyo. Claudel y a failli perdre la vie. Notons que ses premières impressions ont été établies de façon fortement différente dans L'Oiseau noir dans le soleil levant et dans un autre texte, une dépêche publiée en 1963 à l'occasion de l'exposition «Diplomates écrivains ». Dans l'édition critique de L'Oiseau noir, Henri Micciollo définit le poème-reportage intitulé $\grave{A}$ travers les villes en flammes comme " d'abord une composition qui vise à des fins esthétiques $»^{22}$, car Claudel n'a retenu que les événements du $1^{\text {er }}$ et du 2 septembre avec une chronologie bousculée, tout en laissant de côté ce qui s'est passé ensuite.

18 Paul Claudel, Euvre en prose, op. cit., p. 1081-1082.

19 Paul Claudel, Euvre poétique, op. cit., p. 76.

20 Ibid., p. 116.

21 Ibid., p. 81-82.

22 Henri Micciollo, L'Oiseau noir dans le soleil levant de Paul Claudel. Introduction, variantes et notes, op. cit., p. 147. 
Ce qui est à remarquer en premier, c'est que le halo de mystère relié aux flammes dont nous avons parlé blêmit dans ce texte. Et, loin des flammes dramatiques qui " préparent une résurrection ${ }^{23}$, celles-ci contiennent une valeur réaliste : " [...] les incendies ont commencé, de toutes parts les colonnes de fumée s'élèvent, les voies d'eau sont coupées [...] bientôt le quartier de Kanda où j'habite [...] est en feu "; "dans l'intervalle, sur une étendue de je ne sais combien de kilomètres carrés, tout brûle! Une vapeur ardente flotte sur cette cuve qu'attisent encore, par bouffées véhémentes, les derniers souffles du typhon qui expire. De temps en temps, une détonation, une flamme immense qui monte au ciel : c'est un gazomètre qui saute, un dépôt de produits chimiques qui vient d'être touché. ${ }^{24}$ Claudel n'exagère pas les dégâts causés par l'incendie : selon l'extrait de la revue Japon et ExtrêmeOrient de décembre 1923, "l'incendie fit d'impressionnants ravages, sur 95000 maisons de Yokohama, plus de 70000 ont été détruites, le nombre des morts est de plusieurs dizaines de milliers, quant à Tokyo, le pourcentage des bâtiments détruits est de $75 \%$, et on compte plus de 85000 morts dans les quartiers les plus atteints par l'incendie $»^{25}$.

Si la force funeste et irrésistible de la nature, en partie symbolisée par les flammes, est, d'après Claudel, la cause principale du malheur, celles des violences humaines ont néanmoins été complètement voilées dans l'écrit de l'ambassadeur-poète. Le massacre raciste de Coréens dont des estimations officieuses font état d'un bilan de plusieurs milliers à 10000 morts donne un exemple. ${ }^{26}$ Mais aucune trace de cette page obscure de l'histoire du peuple japonais n'a été trouvée dans le récit claudélien. La mer des flammes, n'ayant pas amplifié l'horreur de l'événement, sert pourtant, comme toile de fond du panorama, à observer avec sympathie les survivants :

Le cri des victimes ensevelies sous les ruines n'était pas cet appel impérieux : "Au secours, par ici ! ", mais une modeste supplication : «Dosô, dosô, dosô !" (s'il vous plaît!).

On peut lire aussi une observation plus spécifique :

Un de mes collègues me racontait qu'il avait fait un voyage de nuit entre Yokosuka et Kamakura avec un officier de marine qui allait à la recherche de sa femme et de son fils unique. Une fois arrivé à l'hôtel, il voit peu de temps

23 Gilbert Viprey, «Images de la mort », in Jacques Petit (éd.), Paul Claudel, 3 : Thèmes et images, op. cit., p. 30.

24 Paul Claudel, Connaissance de l'Est, suivi de L'Oiseau noir dans le soleil levant, Paris, Gallimard, 2010, p. 189-191.

25 Henri Micciollo, L'Oiseau noir dans le soleil levant de Paul Claudel. Introduction, variantes et notes, op. cit., p. 152.

26 "Les massacres de Coréens après le séisme de Kanto en 1923 ", in l'Association d'amitié franco-coréenne-Comité Bourgogne [en ligne], http://www.aafc-bourgogne.org/2015/09/lesmassacres-de-coreens-apres-le-seisme-de-kanto-en-1923.html (page consultée le 8 mars 2015). 
après revenir l'officier, la mine parfaitement calme et sereine. Il lui demande des nouvelles de sa famille : "Oh ! tous deux sont morts ", et il se mêle à la conversation générale. Un instant après seulement il remarque : « Excusez-moi si je vous réponds de travers, mais je suis un peu nerveux $»{ }^{27}$

Force est de constater que les réactions des Japonais vis-à-vis de la mort pourraient étonner Claudel car il a noté dans son journal une vingtaine d'années plus tard : " [...] Puis l'étudiante japonaise catholique Katuoka, qui me parle de la mort probable de ses parents en éclatant de rire ${ }^{28}{ }^{8} \mathrm{Ce}$ "stoïcisme japonais ", terme employé par Claudel, qui a dû éprouver deux fois dans les deux ans suivant son arrivée de violentes secousses endommageant l'Ambassade de France à Tokyo ${ }^{29}$, est donc une façon de s'accommoder des circonstances de l'existence, car " [1]e Japon, est plus qu'aucune autre partie de la planète, un pays de danger et d'alerte, continuelle, toujours exposé à quelque catastrophe : raz de marée, cyclone, éruption, tremblement de terre, incendie, inondation. Son sol n'a aucune solidité $"^{30}$. Autrement dit, pour un pays que fréquente la mort brutale et inattendue, la considération principale de l'écrivain au sujet de la vie et de la mort se lie moins à l'espace comme dans le rite, qu'au temps où passe l'être éphémère.

Une autre raison non sans importance pour ce passage est le vieillissement de Claudel. Loin du jeune consul en Chine, le "vieil ambassadeur » de " [1]a maison du pont-des-faisans $»^{31}$, fin 1926, a été très préoccupé par la vieillesse et la mort. Son journal nous montre les témoignages de cet état d'esprit : « Je suis en pourparlers avec la mort, je pèse ses propositions " ${ }^{32}$; "Soixante ans! redressons-nous et rectifions notre tenue avant de pénétrer dans cette froide et solennelle enceinte. ${ }^{33}$ Nous pouvons penser que c'est sans doute par ces deux motifs que Claudel met de loin en loin les Japonais individualisés, et essaie de comprendre l'appréhension de l'être éphémère dans "l'âme japonaise " ${ }^{34}$ à partir de son reflet le plus approprié : l'art et la poésie.

\section{Goutte d'eau éphémère, goutte d'eau éternelle}

Claudel a ainsi conclu, dans un petit poème imitant le haï-kaï japonais, sa vision de la nuit du $1^{\text {er }}$ septembre 1923, en traversant les ruines où s'amoncellent les morts :

27 Paul Claudel, Connaissance de l'Est, suivi de L'Oiseau noir dans le soleil levant, op. cit., p. 186.

28 Paul Claudel, Journal II, Paris, Gallimard, 1969, p. 574.

29 Paul Claudel, Connaissance de l'Est, suivi de L'Oiseau noir dans le soleil levant, op. cit., p. 187.

30 Ibid., p. 184.

31 Ibid., p. 160.

32 Paul Claudel, Journal II, op. cit., p. 732.

33 Ibid., p. 737.

34 Paul Claudel, Connaissance de l'Est, suivi de L'Oiseau noir dans le soleil levant, op. cit., p. 161. 
À ma droite et à ma gauche il y a une ville qui brûle mais la Lune entre les nuages est comme sept femmes blanches.

La tête sur un rail mon corps est mêlé au corps de la terre qui frémit. J'écoute la dernière cigale.

Sur la mer sept syllabes de lumière une seule goutte de lait. ${ }^{35}$

Parmi les thèmes qui évoquent déjà avec évidence la mort (thème de la cigale), nous arrêtons notre regard sur l'image qui termine le poème : une " goutte de lait» dans la mer sous le rayonnement du soleil. En effet, au sujet de la lumière, nous avons des interprétations différentes. Certains commentateurs pensent que c'est le reflet de la lune, blanc, opposé à la lumière (rouge, orange) de la ville qui brûle; d'autres pensent que cela désigne plutôt les rayons du soleil, sources d'origine de la lumière de la lune, parce que les syllabes de lumière évoquent au fond le spectre solaire. Notre travail se situe dans la deuxième lignée. La mer, que ce soit dans le contexte occidental ou dans le contexte oriental, contient en quelque sorte le sens du terme de la vie. Donc quel est le sens de "la lumière " sur la mer, et sa liaison avec cette "goutte de lait " ? En effet, avec une petite nuance, l'image de la "goutte d'eau » se répète vers la fin de son recueil de haï-kai composés au Japon, Cent phrases pour éventail:

Phrase 153 : «Départ/La goutte d'eau à l'extrémité de cette aiguille de pin prête à se réunir à la mer tremble hésite "; phrase 156 : "Un pin la mer il a plu/Loin de tout regard humain la mer est occupée à faire le siège d'une goutte d'eau ${ }^{36}$; phrase $157:$ : Il a plu/un rayon de soleil le lac reflète un pin tout revêtu de gouttes d'eau " ${ }^{37}$; phrase 166 : "La goutte d'eau/sent que toute la mer est occupée à la solliciter $»^{38}$.

D'évidence la " goutte d'eau " est fortement liée aux autres réalités de la nature sur le plan matériel. Le pin et la mer, éléments les plus répétés, indiquant respectivement la plante sur le continent et la destination de l'eau, sont associés aux phénomènes de la pluie et du rayon du soleil. Le lac pourrait être considéré comme un court séjour de l'eau, comme dans d'autres phrases où se montre le torrent qui prépare sa rentrée, comme dans la phrase 93 par exemple : "J'écoute/le torrent qui se précipite vers sa source ". Une telle alliance d'images comprend presque le cycle complet de l'eau dans la nature, et nous fait penser naturellement à la grande circulation atmosphérique de notre planète : l'eau, en tant que liquide, s'évapore en gaz au cours du rayonnement du soleil, tombe de nouveau du ciel en pluie ou en neige, et revient à la mer en coulant sur le continent. Dans ce sens, la "goutte d'eau " passagère n'a jamais une existence définitive, mais des apparences différentes en fonction de ses transformations circulaires. De plus, superposée à la goutte

35 Ibid., p. 198.

36 Paul Claudel, Euvre poétique, op. cit., p. 740.

37 Ibid., p. 741.

38 Ibid., p. 743. 
d'eau matérielle, il y a aussi la goutte d'eau spirituelle. Dans la phrase 163 : "Cil oreille/mots mouillés dont la secrète sensibilité a pour centre une goutte d'eau ${ }^{39}$; phrase 170 : «Dieu une seconde a trouvé cette goutte d'eau au fond de mon âme $»^{40}$. Les âmes, qui portent le sens de la goutte d'eau à l'intérieur, ne jouent alors que ses apparences avec l'écoulement du temps. Le temps, dont la signification n'est pas toujours la même chez Claudel, indique ici plutôt l'alternance saisonnière, non seulement parce que la chaleur et le froid qui décident de la substance de l'eau sont étroitement liés aux saisons, mais aussi pour la forte impression saisonnière tout au long des Cent Phrases.

D'où naît l'éternité dans le monde instantané : les quatre saisons, en se succédant l'une à l'autre dans un ordre temporel, forment un cercle roulant vers l'éternité, sur laquelle la vie et la mort fusionnent en Un. Ce n'est ni « la vie future " " en vide ", ni la " conjonction de deux principes opposés » dont nous avons parlé, mais une sorte d' "harmonie universelle ", l'idée centrale de la belle prose de Tchouang-tseu, livre de chevet pendant le séjour de Claudel au Japon :

C'est un point, d'où ceci et cela, oui et non, paraissent encore non distingués. Ce point est le pivot de la norme. C'est le centre immobile d'une circonférence, sur le contour de laquelle roulent toutes les contingences, les distinctions et les individualités; d'où l'on ne voit qu'un infini, qui n'est ni ceci ni cela, ni oui ni non. Tout voir, dans l'unité primordiale non encore différenciée, ou d'une distance telle que tout se fond en un, voilà la vraie intelligence. ${ }^{41}$

C’est par cette éternité que se fixe la joie de vivre des êtres éphémères dans Cent Phrases; c'est par cette éternité que la " goutte de lait » dans la nuit du $1^{\text {er }}$ septembre 1923 attend sa prochaine évaporation sous les "sept syllabes de lumière "; c'est sans doute par cette éternité que le poète de "Maturité " chante à l'automne de l'année 1937 ainsi que de sa propre vie : "Tout ce qui passe est passé/Eh bien, on va recommencer. ${ }^{42}$

39 Ibid., p. 742.

40 Ibid., p. 744.

41 Tchoang-tzeu, "Harmonie universelle ", dans Wikisource la bibliothèque libre [en ligne], https://fr.wikisource.org/wiki/\%C5\%92uvre_de_Tchoang-tzeu/Chapitre_2._Harmonie_ universelle (page consultée le 20 octobre 2019).

42 Paul Claudel, Euvre poétique, op. cit., p. 908. 
Quelle que soit la valeur de la sagesse orientale inspirant ces textes, la mort est, en fin de compte, "comme une fin que Dieu impose à l'homme » et " une délivrance de l'ignorance de la source ${ }^{43}$ dans la vision chrétienne de Claudel. Or, le dialogue avec l'Extrême-Orient a non seulement ensoleillé son regard envers la mort, mais l'a aussi enrichi au sens métaphysique et artistique.

Xu Yi

Université de Wuhan

Université Bordeaux Montaigne

TELEM EA 4195

yi.xu@etu.u-bordeaux-montaigne.fr

\title{
Résumé
}

Tout en gardant une place primordiale, la question de la mort chez Paul Claudel a connu une série de nuances durant ses séjours en Chine et au Japon. La perception culturelle, la familiarisation avec la littérature, l'emprunt artistique et la réflexion philosophique ont contribué à créer son propre art de l'« être-vers-la-mort».

\section{Mots-clés}

Paul Claudel, vie, mort, Chine, Japon.

\begin{abstract}
The question of death in Paul Claudel's writing career has experienced a series of changes during his trip from China to Japan while retaining its primordial place. Cultural perception, familiarization with literature, artistic borrowing and philosophical thinking help to create his own art of "being-towards-death".
\end{abstract}

\section{Keywords}

Paul Claudel, life, death, China, Japan.

43 Bei Huang, Segalen et Claudel: Dialogue à travers la peinture extrême-orientale, Rennes, Presses Universitaires de Rennes, 2007, p. 406. 